\title{
The Study on Revelation of Glaucoma using Structural Features
}

\author{
V. R. Balaji and C. Thulasi \\ Department of Electronics and Communication Engineering, Sri Krishna College of Engineering and Technology, \\ Coimbatore - 641008, Tamilnadu, India; balajivr@skcet.ac.in, thulasichokkalingam@gmail.com
}

\begin{abstract}
Objectives: To find the presence of glaucoma disease in eyes. It has to be found at the earlier stages; otherwise it will cause permanent damage to eyes. Methods/Statistical Analysis: Fundoscopy is one of the methods available to find the glaucoma disease. This is based on biomedical technique which sometimes may not able to reveal the presence of glaucoma. So a new unique method has to generated. By using hybrid feature set from the digitalized fundus images, it can be easily detected. Findings: The glaucoma disease is one which affects millions of people across the world as found by WHO. Worldwide, the number of people with glaucoma was estimated at over 64.3 million in 2013, and is expected to increase to 76.0 million in 2020. The eye needs a certain amount of pressure to keep the eyeball in shape so that it can work properly. In some people, the damage is caused by raised eye pressure. Others may have an eye pressure within normal limits but damage occurs because there is a weakness in the optic nerve. In most cases both factors are involved but to a varying extent. Application/Improvements: The structural features taken from the image can help us to improve the findings of the glaucoma disease. The parameters will give complete information on the impact on the eyes. These details will help us in detecting the disease at a earlier stage than at a later stage.
\end{abstract}

Keywords: Glaucoma, Histogram, Principal Component Analysis, Structural Features

\section{Introduction}

Glaucoma is one of the chronic disease which is not detected at an early stage may lead to permanent damage. It affects the optic nerves and will get worse as time proceeds. This is the second type of blindness found across the world as declared by WHO. Millions of people across the world get detected by the disease and it keeps on increasing day by day. Eye disease developing from increasing intravascular pressure caused by tumour, a fluid makes by the attention. Generally eye, a novel value is maintained because the quantity of fluid secreted is adequate to the number of fluid discharged by the attention. It's typically coupled to a buildup of pressure within your eye. Eye disease may be genetic in some cases and will known to people at the last stage. The high pressure cause damage to the optic nerve and it will have impact in the brain. If the injury continues,
Glaucoma will cause permanent vision loss. Glaucoma will cause total permanent visual impairment in long course. Mostly the symptoms such as pain are not shown in eye disease. The application of liquids at the eye may increase the stress and cause higher level of damage to the eye if not medicate properly and cause the patient to loss communication through the eye.

\section{Literature Survey}

The glaucoma detection has to be perfect in every stage to avoid visual damage. Even if undetected will cause severe damage to the eye. In medical imaging, lot of research is going on in this area to develop automatic detection of glaucoma. It is also done in such a way to produce computer aided design (CAD) tool that will help ophthalmologists to diagnose the disease. Various 
methods such as digital image processing, deep learning, artificial Intelligence tools are used to stand out during this analysis sector and are available up with additional correct results that may facilitate in additional help for diagnosis of glaucoma. Various methods are also used for diagnosis. In a study ${ }^{1}$, a deep learning technique is proposed to found the $\mathrm{CNN}$ based identification of neuroretinal rim and optic disc cupping as well as the lamina cribrosa (LC) and its surrounding areas as the regions significantly associated with the glaucoma classification. Local binary pattern and Daugman's algorithm are done to know the details of features. The parameters are compared with Higher Order Spectra $(\mathrm{HOS})^{2}$. In another study ${ }^{\underline{3}}$, a novel algorithm was proposed to find structural and non structural parameters. An automatic method using feature Learning is proposed along with Deep Learning (ALADDIN) in one of the study ${ }^{4}$.

\section{Methodology}

The following detailed procedure was then employed as the proposed system of detecting glaucoma by extracting features using Naive Bayes and classified by Decision Tree algorithm.

Pre-processing may be a common name for operations with images at the bottom level of abstraction both input and output are intensity images 5 . The aim of pre-processing is an improvement of the image data that suppresses unwanted distortions or enhances some image options necessary for additional process.

\subsection{Principal Component Analysis}

In signal processing, Principal Component Analysis is used to delineate into transform for the provided group of $\mathrm{n}$ input vectors. The PCA processing is shown in Figure 1 . The length of the vectors will be denoted by identical length $K$. It can be transformed as a n-dimensional vector

$\mathrm{x}=[\mathrm{x} 1, \mathrm{x} 2, \ldots \mathrm{xn}]$

$\mathrm{y}=\mathrm{A}(\mathrm{x}-\mathrm{mx})$

The vector xis $\mathrm{n}$ dimensional since it contains $\mathrm{n}$ input variables. The dimension of $\mathrm{cx}$ isxn if it is multidimensional. The variances are the individual elements $\mathrm{Cx}(\mathrm{i}, \mathrm{i})$ found in principal diagonal.

$\mathrm{Cx}(\mathrm{i}, \mathrm{i})=\mathrm{E}$

of $x$ and the other values of $\mathrm{Cx}(\mathrm{i}, \mathrm{j})$ indicate the difference ${ }^{6}$ in the input $x i, x j$.

$\mathrm{Cx}(\mathrm{i}, \mathrm{j})=\mathrm{E}$

Between variables of the input $x i, x j$. It shows orthonormal ${ }^{7}$ from the rows of $A$ in equation (1)

PCA is given according to the relation,

$\mathrm{x}=\mathrm{ATy}+\mathrm{mx}$

\subsection{Structural Features}

One of the best approaches for describing texture is to use the statistical moments ${ }^{\underline{8}}$ of the grey level bar graph of the image. The varied statistical features are supported by histogram which is grey level, co-occurrence matrix ${ }^{9}$,

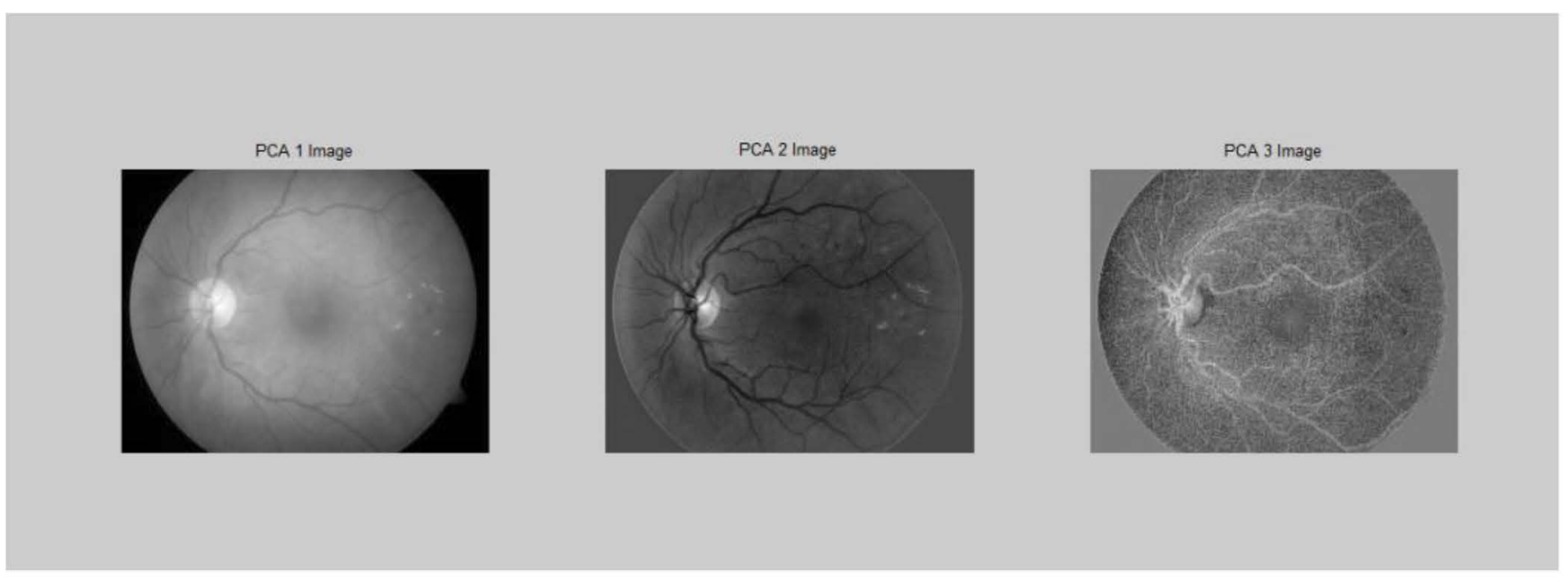

Figure 1. PCA Processing. 
and edge frequency ${ }^{4}$ and run length distribution. The structural features are shown in Figure 2.

\section{- Histogram Based Features}

The histogram-based features employed in this work are 1st order statistics that embrace mean, median and variance. Let $\mathrm{z}$ be a stochastic variable denoting image grey levels and $\mathrm{p}(\mathrm{zi}), \mathrm{i}=0,1,2,3, \ldots \ldots . \mathrm{L}-1$, be the corresponding histogram $\frac{10}{}$, wherever $L$ is that the variety of distinct grey levels. The features are calculated using the above-mentioned histogram 11 .

\section{- Mean}

The mean provides the common grey level of every region and it's helpful solely as a rough plan of intensity not very texture.

$$
\begin{gathered}
\mu=\frac{1}{N} \sum_{i=0}^{N-1} x_{i} \\
m_{a}=\frac{1}{A} \sum_{(m, n) \in R} a[m, n]
\end{gathered}
$$

\section{- Entropy}

In Image, Entropy is outlined ${ }^{12}$ as corresponding states of intensity that individual pixels will adapt. It's employed in the measuring and analysis image details,

$$
H\left(S_{m}\right)=-\sum_{n=1}^{256} P_{n\left(S_{m}\right) \cdot \log 2\left(\left(P_{n}\left(S_{m}\right)\right) \ldots \ldots . . m=1, \ldots . M\right.}
$$

\section{- Standard Deviation}

The standard deviation $\frac{13}{}$ of the unbiased estimate is given by

$$
S_{a}=\sqrt{\frac{1}{A-1}} \sum_{m, n \in R}\left(a[m, n]-m_{a}\right.
$$

\section{Results and Discussion}

The Principal Component Analysis is done to delineate into transform and the parameters are compared with the help of various images. The various images are taken in order to find the minute details involved in the disease. The structural features are taken and compared for various instances. The image is analyzed by taking the parameters such as deviation, maximum pixel, entropy, standard deviation as shown in Figure 3.

\section{Conclusion}

Glaucoma could be serious disease if not detected at proper time and will lead to lot of various complications.



Figure 2. Structural Features. 


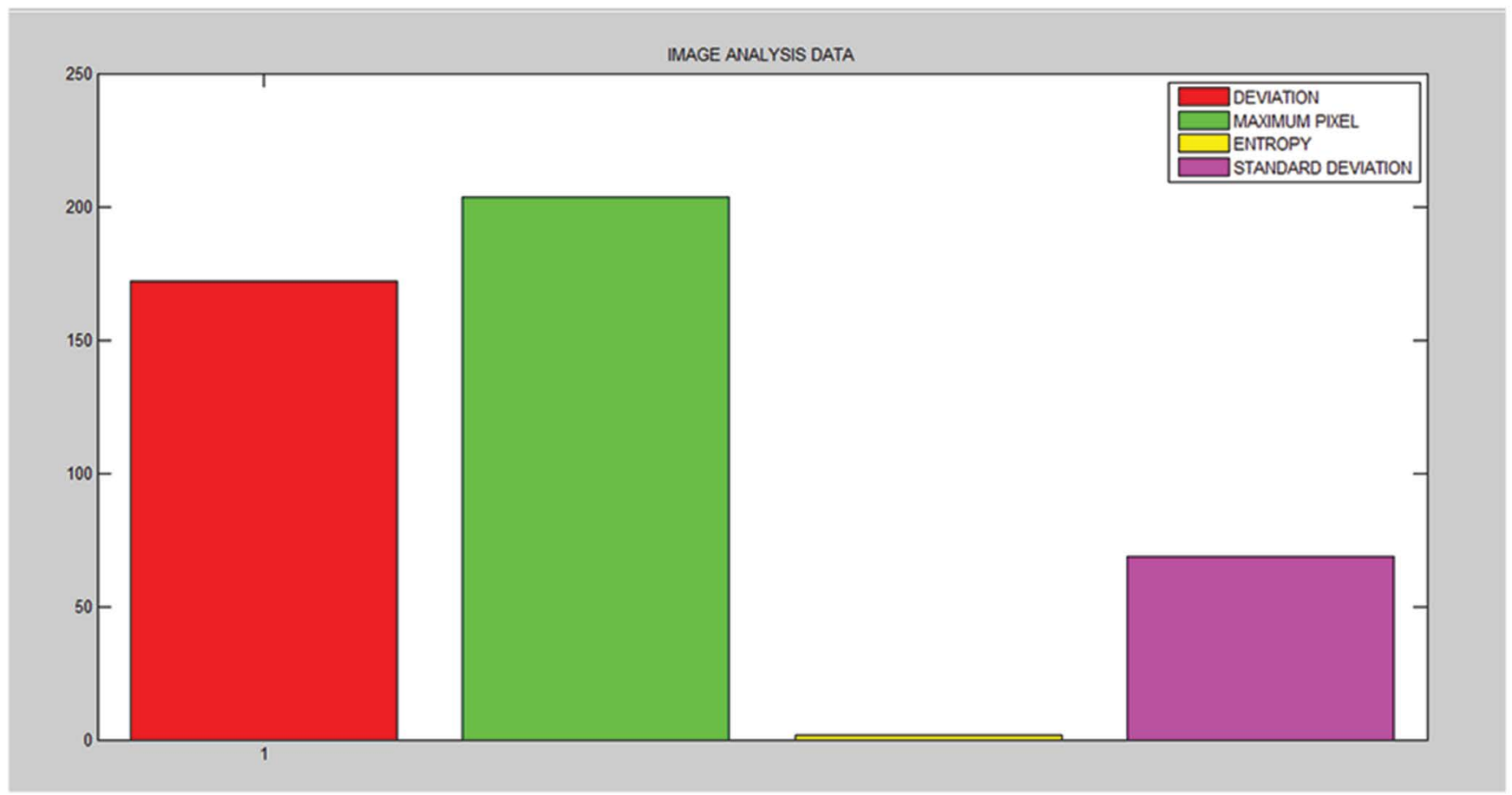

Figure 3. Image Analysis.

Fundoscopy helps eye doctors to research the inner retinal structural layer analysis. This may help to avoid glaucoma to spread across the eyes. The Fundus Image of an eye is analysed by Principal Component Analysis (Preprocessing method) and the image is further analysed to get histogram Equalisation for adjusting pixel values. The image analysis, then the image is converted to RGB Color components for defining the intensities. In the proposed method, structural features such as Maximum Pixel, Standard Deviation, Entropy and Deviation are calculated. In next level, the textural features will be calculated and it can be combined with hybrid structural features. Finally, the detection of glaucoma can be found out by using decision tree algorithm.

\section{References}

1. Maetschke S, Antony B, Ishikawa H, Wollstein G, Schuman JS, Garvani R. A feature agnostic approach for glaucoma detection in OCT volumes. arXiv preprint, Elseiver. 2018; p. 1-13.

2. Ragavendra U, Gudigar A, Bandary SV. Deep Convolution Neural Network for Accurate Diagnosis of Glaucoma Using DigitalFundusImages. InformationSciences.2018;441:41-9. https://doi.org/10.1016/j.ins.2018.01.051
3. Abbas Q. Glaucoma-Deep: Detection of Glaucoma Eye Disease on Retinal Fundus Images using Deep Learning. International Journal of Advanced Computer Science and Applications. 2017; 8(6):1-5. https://doi.org/10.14569/IJACSA.2017.080606

4. Balaji VR. A Comparison of Compression Sensing Algorithm and DUET algorithm for Advanced DCT based Speech Enhancement System for Vehicular noise. International Journal of Pure and Applied Mathematics. 2018; 119(12):1385- 94.

5. Borgalli RA, Gautam HP, Parayil WG. Automated Glaucoma Detection Techniques Using Fundus Image. International Journal Of Technology Enhancements And Emerging Engineering Research. 2015; 3(12):1-8.

6. Quan Y, Cheng J, Lee BH, Yow AP, Wong DWK, Automatic Glaucoma Screening Hybrid Cloud System with Pattern Classification Algorithms. IEEE 2nd International Conference on Signal and Image Processing. 2017; p. 21922.

7. Sakthivel K, Narayanan R. An automated detection of glaucoma using histogram features. International Journal of Ophthalmology. 2015; 8(1):194-200. PMid:25709931 PMCid:PMC4325265

8. Narasimha-Iyer H, Roysam BCA, Stewart CV, TanenBaum HL, Singh HMA. Robust detection and classification of longitudinal changes in color retinal fundus images for monitoring diabetic retinopathy. IEEE Transactions on 
Biomedical Engineering. 2006; 53(6):1084-98. https://doi. org/10.1109/TBME.2005.863971 PMid:16761836

9. Balaji VR. A discrete fractional cosine transform based speech enhancement system through Adaptive Kalman filter Combined with perceptual weighting filter with pitch synchronous analysis. International Review on Computers and Software. 2013; 8(9):2288-95.

10. Wang J, Chen C, Li F, Wang Z. S-D Net: Joint Segmentation and Diagnosis Revealing the Diagnostic Significance of Using Entire RNFL Thickness in Glaucoma. 1st Conference on Medical Imaging with Deep Learning (MIDL). 2018; p. $1-10$.
11. Jose AM, Balakrishnan AA. A Novel Method for Glaucoma Detection Using Optic Disc and Cup Segmentation in Digital Retinal Fundus Images. International Conference on Circuit, Power and Computing Technologies [ICCPCT] . 2015; p. 1-5. https://doi.org/10.1109/ICCPCT.2015.7159441

12. Burgansky-Eliash Z, Wollstein G, Bilonick RA, Ishikawa H, Kagemann L, Schuman JS. Glaucoma detection with the Heidelberg Retina Tomograph (HRT). Ophthalmology. 2007; 114(3):466-71. https://doi.org/10.1016/j.ophtha.2006.08.022 PMid:17141321 PMCid:PMC1945822

13. Gonzalez RC, Woods RE. Digital Image Processing using MATLAB, 2nd Edition. Prentice Hall. 2002; p. 1-827. 